\title{
No to Uncertainty of Medicine by People: A New Gathering Storm
}

\author{
Emran Bin Yunus ${ }^{1 *}$ \\ 'Head of Nephrology \& Principal (Former) \\ Chittagong Medical College \\ Chittagong, Bangladesh \\ \& \\ GCP Alumni, TDR, WHO.
}

${ }^{*}$ Correspondence to:

\section{Professor Emran Bin Yunus}

Internist \& Nephrologist

CSCR (Pvt) Ltd

1675/A, O R Nizam Road

Chittagong, Bangladesh.

Mobile : +8801711748746

Email : ebyunus@yahoo.com

www.banglajol.info/index.php/CMOSHMCJ
Medicine is a discipline of uncertainty. In diagnosing and managing diseases doctors try to match their knowledge with that of the presenting scenario of the patient. It's like that of putting a peg in to a hole, round peg to a round hole or a square one and various composition ${ }^{1}$. Medical diagnostic process is a deductive affair with a function of unknown by known. Most doctors don't acknowledge this phenomenon actively. This is the impact of medical course training and blinding of gathered knowledge, skill and attitude. The accumulated experience and evidence provide doctor the edge to decide pattern of peg for hole or to customise. But there remains uncertainty. When patient want sure diagnosis and confirmed cure doctor cannot provide always, the bad side is that doctor is not acknowledging that inability. For example, we may pick up the most simple and common problem, fever without focal signs. Even with a focal sign for example pneumonia the scenario is not changed much. The certainty versus uncertainty remains.

The traditional expectations of patients are: patient wants to be listened to and be understood, patient wants to be treated as fellow human being, patient wants reasonable degree of knowledge and skill in caring doctor, patient wants to be reasonably be informed and, patient wants whatever the reason not to be abandoned ${ }^{2}$.These sound so naive but if one critically analyse shall find how difficult to fulfil these expectations and by passage of time it is becoming more and more twisting.

All patients have one issue in common that is they want cure. All doctors have in contrast the common issue patients are cases for applying diagnostic and management deductions. Doctors are shy or reluctant to expose the uncertainty of the ongoing outcome and finale. This is a great divide between doctors and patients in behalf of diagnosis and management and outcome ${ }^{3}$.

The fluid patient perceptions and ideas are being continually funnelled with consolidation in the direction of nothing but cure by communication boom, media dissemination and also by doctors themselves. These are mostly on pros side and not on cons ${ }^{4}$. The result is the progressive raising the expectations of patients and their kith and kin. It has been so high set that even a moribund patient like terminal state with cancer is put to life support and may also for dialysis, and when patient succumbs there are commotions, vandalisms, blaming doctors, media upbeating and law enforcer interventions ${ }^{5}$. In absence of guidelines every group concerned reaps from the situation. This is a vicious cycle increasing day by day ${ }^{6}$. 
Our curriculum imparts us the process of collecting history, symptoms and signs funnel to compiling in to a diagnosis and some differential diagnoses with proceeding to tests. We fail to recognise the cognitive bias in this process. Defying this we learn to convert the grey scale narration of patient into a black and white spectacle categorised to neat diagnosis and label. The whole process is an obsession. The limitation of black and white approach is not made a practice and culture to the patients. The uncertainty principle is not only in clinical sciences but also in all disciplines. This implies that science is not the whole truth rather a partial truth for particular period and space. If one divides 10 by 3 one cannot get concrete answer without a grey area.

Patients die off medicines, procedures, manoeuvres and other likes. Most of them are recognized standard Severe Adverse or Adverse Events. These are uncertainty and by current professional knowledge base most of these are acceptable. It is not so to patient and people. Unlike doctors they want 'what' and not 'how' and 'why'. In fact in the process of management 'what' has been in principle and practice is dynamic 'hypothesis' and not a fixed diagnosis. Medical Curriculum expands through formal, nonformal and hidden attributes. There are no attributes to educate doctors to acknowledge the deficit in pacifying the 'what' or 'diagnosis'?

Now when doctors are shy and people are charged and communication media are puffing the scenario has been reshaping by undermining of professional ground and perception of people. The most unwelcome outcome that is occurring is the loss of trust of people ${ }^{8}$. Nothing but cure now the only acceptable demanding expectation of them.

Down the time line of 21 century the issue will be progressively more and more complicated. The doctors and producers of doctors especially in Bangladesh are seemingly ignoring the evolving situation of this growing incongruence between certainty and uncertainty of medicine in other word trust of patients on doctors. People are rejecting the uncertainty principle of medicine and a new storm has been gathering in this behalf and is growing exponentially. Doctors of Bangladesh should now develop innovative learning and practice model to be idols to the future doctors and patients with breaking the shyness of telling the truth that medicine is a science and discipline of uncertainty. Per Osler it is, 'Medicine is a science of uncertainty and art of provability'. Uncertainty was in yesterday, is now in today and shall be in tomorrow. A former editor of 'The New England Journal of Medicine', Dr Joseph Garland recalled the advice given by the 'autocrat of the breakfast table' to the younger doctors, 'Don't dabble in the muddy sewer of politics, nor linger by the enchanted stream of literatures, nor dig in the far off fields of alien sciences for hidden waters, the great practitioners are those who concentrate all their powers on their business' ${ }^{9}$. This advice is no longer tenable the earlier it is captured in text and spirit by the doctors the better and safer shall be the professional roadmap in the Odyssey of uncertainty of medicine there by diffusing the gathering storm.

\section{DISCLOSURE}

The author declared no competing interest.

\section{REFERENCES}

1. Saint S, Saha S, Tiemey LM: A Square Peg in a Round hole. N Engl J Med. 1998;338:379-383.

2. Smith LH: Medicine as an art. In Wyngaarden JB, Smith LH, Benett JC (Eds): Cecil Text Book of Medicine. WB Saunders, 19th Edition. 1992;6-7.

3. Kassirer JP: Our Stubborn Quest for Diagnostic Certainty: A Cause of Excessive Testing. N Engl J Med. 1989;320:1489-1491.

4. Staniszewska S, Ahmed L: Patient expectations and satisfaction with health care. Nursing Standard. 1998;12(18):34-38.

5. Ornstein C: Conflicts between patients and doctors spread onto social media. The Portland Press Herald. May 28, 2016.

6. Johnson T: Shattuck Lecture - Medicine And The Media. The N Engl J Med. 1998;339:87-92.

7. Simpkin Al, Schwartzstein RM: Tolerating Uncertainty - The Next Medical Revolution. N Engl J Med. 2016;375:1713-1715.

8. Blendon RJ, Benson JM, Hero JO: Public Trust in Physicians - US Medicine in International Perspective. N Engl J Med. 2014;371:1570-1572.

9. Lister J: Shattuck Lecture - The Politics of Medicine in Britain and The United States. N Engl J Medicine. 1986;315:169-174. 